\title{
Role of microRNAs in Vascular Remodeling
}

\author{
Y.-C. Fang ${ }^{1}$ and C.-H. Yeh ${ }^{*}, 1,2$ \\ ${ }^{1}$ Division of Thoracic and Cardiovascular Surgery, Chang Gung Memorial Hospital, Keelung, Taiwan \\ ${ }^{2}$ College of Medicine, Chang Gung University, Tao-Yuan, Taiwan
}

\begin{abstract}
Besides being involved in the gradual formation of blood vessels during embryonic development, vascular remodeling also contributes to the progression of various cardiovascular diseases, such as; myocardial infarction, heart failure, atherosclerosis, pulmonary artery hypertension, restenosis, aneurysm, etc. The integrated mechanisms; proliferation of medial smooth muscle cell, dysregulation of intimal endothelial cell, activation of adventitial fibroblast, inflammation of macrophage, and the participation of extracellular matrix proteins are important factors in vascular remodeling. In the recent studies, microRNAs (miRs) have been shown to be expressed in all of these cell-types and play important roles in the mechanisms of vascular remodeling. Therefore, some miRs may be involved in prevention and others in the aggravation of the vascular lesions. miRs are small,

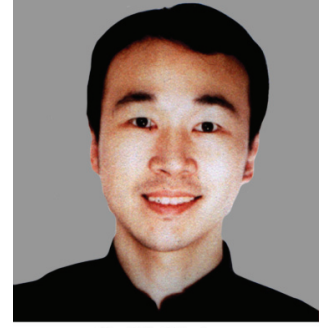

C.-H. Yeh endogenous, conserved, single-stranded, non-coding RNAs; which degrade target RNAs or inhibit translation post-transcriptionally. In this paper, we reviewed the function and mechanisms of miRs, which are highly expressed in various cells types, especially endothelial and smooth muscle cells, which are closely involved in the process of vascular remodeling. We also assess the functions of these miRs in the hope that they may provide new possibilities of diagnosis and treatment choices for the related diseases.
\end{abstract}

Keywords: Cardiovascular disease, endothelial cell, macrophage, microRNA, proliferation, smooth muscle cell.

\section{INTRODUCTION}

Vascular remodeling during human embryonic development may occur physiologically, through processes such as angiogenesis or arteriogenesis [1] or pathologically, through injury of vascular cell walls, as in the case of vascular disease [2]. Pulmonary artery hypertension, one of the vascular diseases, is caused by functional and structural changes in the pulmonary artery, which leads to the thickening of cell walls and an increase in the resistance of circulating blood. These processes can lead to further complications such as the dysfunction of endothelial cells, activation of fibroblasts and smooth muscle cells, the crosstalk between these cells, and the involvement of blood cells [3]. These cells are also involved in the process of atherosclerosis, which is caused by the activation of circulating immune cells and the dysfunction of lipid metabolism [4]. Immune cells, including monocytes and macrophages, recruit endothelial cells, which cause inflammation, to infiltrate and activate smooth muscle cells leading to thickening of the blood cell walls [5]. Vascular remodeling mainly involves the contribution of four cell types: the fibroblasts in the adventitial layer, the smooth muscle cells in the medial layer, the endothelial cells in the intimal layer, and the macrophages in the blood stream [6]. Firstly, the

*Address correspondence to this author at the Department of Thoracic \& Cardiovascular Surgery, Chang Gung Memorial Hospital at Keelung, 222 Mai-Chin Road, Keelung, 204, Taiwan; Tel: 886-2-24313131, Ext. 2625; Fax: 886-2-24332655;

E-mail: yehccl@cgmh.org.tw activation of the fibroblasts in the adventitial layer releases the platelet-derived growth factor, endothelinI, or serotonin, etc. This stimulates the migration and proliferation of fibroblasts and smooth muscle cells and thickening of the cell walls, increasing susceptibility to cardiovascular diseases [7]. Other molecules also contribute to the process of cell wall thickening [8]. The transforming growth factor will stimulate the differentiation of fibroblasts into myofibroblasts, which not only infiltrate the medial layer to cause thickening but also allow the deposition of extracellular matrix protein [9]. The transforming growth factor and cytokines also translocate into the medial layer, inducing migration, proliferation, contraction, and resistance to apoptosis in smooth muscle cells. In short, the infiltration of the fibroblasts will increase the proliferation of smooth muscle cells resulting in an increased thickness of the cell walls [10]. Secondly, the thickness of intimal layer is primarily regulated by the proliferation of intimal cells and the growth of endothelial cells results in cellular dysfunction. Activated endothelial cells will release endothelin-1 to influence the contraction of blood vessels, elastase to deposit extracellular matrix metalloproteinase and decrease the elasticity of blood cells, and tenascin- $C$ to induce the proliferation of smooth muscle cells. The function of miRs in these cells, however, is not yet clear. Thirdly, the main reason for the increased thickness of cell walls is the proliferation of smooth muscle cells. There are two kinds of phenotypes produced from environmental stimuli: one is the phenotype of contraction (differentiation) and the other is the synthesis (proliferation) [11]. At each different 
stage of development, smooth muscle cells express varied phenotypes as they progress from embryonic to adult stage. At the adult stage, in the case of vascular disease, the phenotype changes from a contractile state to a synthetic state. In this situation, the levels of contractile proteins will decrease while cell proliferation increases. Some studies have also shown that, in the modeling process, the transformation of smooth muscle cells was regulated by miRs, which has also been shown in other cells. Therefore, we reviewed the miRs which were related to vascular remodeling. It has been highlighted that, while one miR can control and regulate the expression of many genes, conversely, one mRNA may also be regulated by several different miRs. Smooth muscle cells are the main cell-type involved in proliferation. Injuries involving the growth factors or vascular vessels may induce progression from a contractile or differentiated state into a proliferative or synthetic state. Persistence of this proliferative state will lead to the induction of vascular remodeling [12].

\section{miR-1}

miR-1 is an miR which is found in the skeletal muscle and smooth muscle cells. It is closely related to the physical and pathological processes of vascular modeling, including heart development, arrhythmias, ischemia, myocardial infarction and cardiac hypertrophy [13]. Some studies have shown that the expression of miR-1 is down-regulated in cardiac hypertrophy and chronic myocardial infarction and speculate that miR-1 may inhibit cell proliferation and hypertrophy $[14,15]$. However, in other cardiovascular experimental models, such as ischemic preconditioning, ischemia/reperfusion, acute myocardial infarction, oxidative stress, and hyperglycemia, the expression of miR-1 is up-regulated [16, 17]. Therefore, miR-1 could be an activator of these diseases. Some researchers have shown the mechanisms of action of miR-1 on vascular diseases to further clarify the function of miR1 according to the size of heart or hypertrophy, the contractile function, and the conductance of heart.

Zao et al. reported that miR-1 targets the transcription factor Hand2, which increases the proliferation of ventricular cardiomyocytes, miR-1 was suggested to prevent vascular injuries by decreasing the thickness of cell walls [18] (Fig. 1). In other animal models, miR-1 also plays a protective role and has been linked to hypertrophy. In the model of functional overload and trans-aortic constriction (TAC) induced hypertrophy, the expression of miR-1 is down-regulated to half through binding of the 3'UTR of target genes, cyclin dependent kinase 9, Ras GTPase-activating protein, fibronectin, and Ras-homolog enriched in the brain [14], which was closely linked to the regulation of genes related to calcium. Some studies reported miR1 -induced down regulation of the expression of the transcription factors, mef2a and gata4, which are related to calmodulin and cardiac hypertrophy along with myocardial infarction. Inhibition of these target genes is an ideal way to treat cardiac hypertrophy.
Furthermore, miR-1 inhibits cardiac hypertrophy thus protecting the heart from hypertrophy-related injury [19] and preventing the expression of calmodulin. Elia et al. report IGF-1 as the target gene of miR-1 [20]. They summarized that miR-1 expression was related to the level of IGF-1 in animal models of cardiac hypertrophy, cardiac mass and thickness in patients.

miR-1 may also affect cytoskeleton regulatory proteins to further regulate hypertrophy [15]. Overexpression of miR-1 targets the 3'UTR of twinfilin-1 mRNA directly to mitigate the hypertrophy, while downregulating endogenous twinfilin-1 [15]. Parallel to regulating hypertrophy, miR-1 is also involved in the contractile functioning of the heart [21]. Jiang et al. concluded that miR-1 could inhibit the contraction of smooth muscle cells thus causing heart failure [21]. Furthermore, some studies described that miR-1 inhibited the expression of MYLK3, CALM1, and CALM2 directly, which in turn led to down-regulation in the expression of CaM and CMLCK, followed by downregulation of phosphorylation of MLC2v or CaMKIID-cMyBP-C and impairment of contractile function [17]. While inhibiting hypertrophy or contractile function, mir1 also affects the conductance of the heart. Overexpression of miR-1 may down-regulate $\mathrm{Cx} 43$ to inhibit not hypertrophy, but ventricular tachyarrhythmias and ventricular tachycardia [22]. In summary, miR-1 may attenuate the progression of hypertrophy and prevent the heart from remodeling and developing into the characteristic phenotype of heart failure, but it may also impair the cardiac functions of contraction and conductance.

\section{miR-21}

miR-21 is expressed in fibroblasts [23], cardiomyocytes [24], and endothelial cells [25]. In these cells, the function of miR-21 is to regulate fibrosis in fibroblasts, apoptosis in cardiomyocytes, and cell proliferation, migration, and tubulogenesis in endothelial cells. Knockdown of miR-21 may inhibit cardiac fibrosis and alleviate the detrimental effects of cardiac fibrosis. Therefore, the knockdown of miR-21 is a potential therapeutic strategy for the treatment of cardiac fibrosis [23]. Cardiac fibrosis involves vascular remodeling and is characterized by the proliferation of cardiac fibroblasts and extracellular matrix (ECM) deposition in the myocardial interstitial space. This can impair the correct functioning of heart [26]. Cardin et al. found that over-expression of miR-21 targeted Sprouty1 [27], and Thum et al. also found that it up-regulated the phosphorylation of MAPK inducing increased fibrosis [28] (Fig. 2).

miR-21 plays a role in apoptosis. Cheng et al. utilized adenovirus-mediated gene transfection based overexpression of miR-21 to show that miR-21 can protect the heart from hypoxia/re-oxygenation (H/R)injury by reducing cardiomyoctic apoptosis [24]. Furthermore, Lin et al. found that knockdown of miR-21 enhances $\mathrm{H}_{2} \mathrm{O}_{2}$-induced apoptosis through upregulating the target gene, programmed cell death 4 (PDCD4) [29]. In other cases, over-expression of miR- 


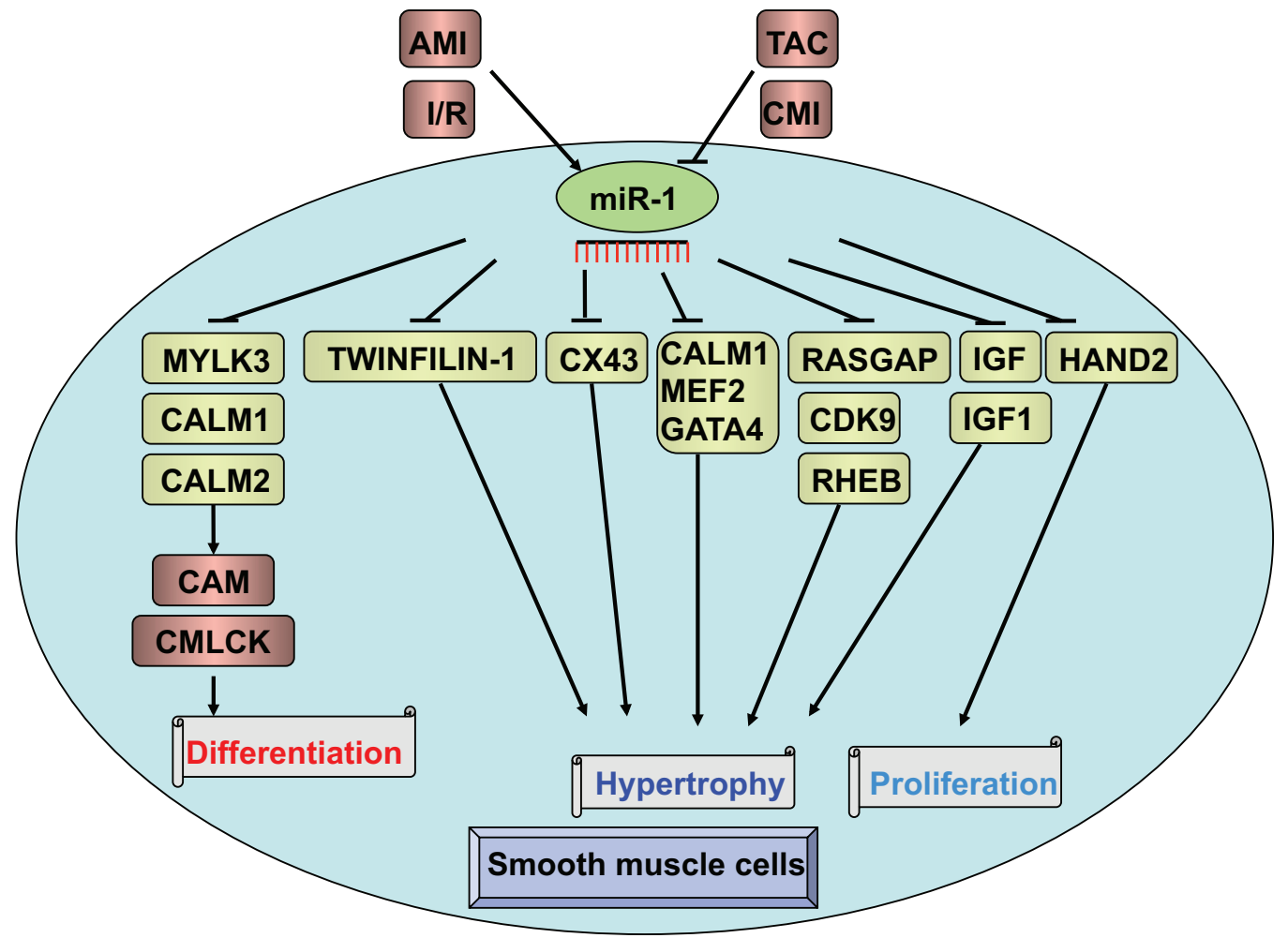

Fig. (1). Modulation of proliferation, hypertrophy, and differentiation by miR-1 in smooth muscle cells. miR-1 inhibited proliferation or hypertrophy via down-regulation of its target genes, such as Hand2, IGF, IGF1, RasGFP, CDK9, Rheb, CALM1, MEF2, GATA4, Cx43, Twinfilin-1, etc. miR-1 inhibited the differentiation of these cells through down-regulation of the expression of MYLK3, CALM1, and CALM2, which in turn inhibited the activity of CaM or CMLCK. Arrows $(\rightarrow)$ represent up-regulation. Capped lines $(-)$ indicate inhibition. Abbreviation: acute myocardial infarction (AMI), calmodulin 1 (CALM1), calmodulin (CaM), cardiac myosin light chain kinase (cMLCK), chronic myocardial infarction (CMI), cyclin dependent kinase 9 (Cdk9), insulin-like growth factor 1 (IGF1), ischemia/reperfusion (I/R), myocyte enhancer factor 2 (Mef2), Ras GTPase-activating protein (RasGAP), Ras homolog enriched in brain (Rheb), trans-aortic constriction (TAC).

21 inhibited these responses [24]. Along with the protective effect of miR-21 through the suppression of PDCD4, over-expression of miR-21 also targets phosphatase and tensin homolog (PTEN), leading to an increase in phosphorylation and activity of AKT, followed by inhibition of apoptosis [30]. The two target genes of miR-21, PDCD4 and PTEN, induce apoptosis [28, 31].

Considering that miR-21 can regulate fibrosis and apoptosis, some researchers also elucidated its role in hypertrophy. Despite the normal phenotype of miR-21null mice, upon exposure to stress, these mice showed increased cardiac hypertrophy and fibrosis compared to wild-type mice. Researchers suggested the knockout of miR-21 caused the hypertrophy [32]. However, Zhang et al. reported that knockdown of miR-21 with an intravenous injection of LNA anti-miR-21 did not result in cardiac hypertrophy [33]. Furthermore, miR-21 may regulate phenotypes in endothelial cells and inhibit proliferation, migration, or tubulogenesis through downregulation of RhoB [34].

\section{miR-29}

The expression of miR-29 family (miR-29a, b, and c) was down-regulated in the border zone of the heart following myocardial infarction [35], implying that it plays protective role in disease progression. Studies have highlighted that miR-29 may degrade certain genes relating to fibrosis such as collagens, elastin, and fibrillins. It has been implied that down-regulation of miR-29 could increase activity of these genes and lead to cardiac fibrosis [36]. The knockdown of miR-29 with antogomirs in vivo and in vitro increased progression of cardiac fibrosis. Higher levels of cardiac fibrosis could enhance cardiac remodeling, resulting in increased thickening of the cell walls and diminished contractile function in the heart [37].

Not only does miR-29b have a protective effect, it may also constitute a possible treatment strategy against hypertensive cardiac fibrosis. Zhang et al. reported inhibition of cardiac fibrosis by miR-29b and improved heart function [33]. They demonstrated that suppression of TGF- $\beta / S$ mad 3 signaling; the upstream molecules of miR-29b, can increase the expression of $\mathrm{miR}-29 \mathrm{~b}$ and inhibit angll-induced cardiac fibrosis. Inhibition of this pathway could represent a potential therapeutic avenue to treat cardiovascular diseases [33] (Fig. 3).

Knockdown of miR-29 with antagomirs increased the expression of myeloid cell leukemia sequence 1 causing inhibition of ischemia/reperfusion-induced cell apoptosis and necrosis, leading to mitigation of cardiac 

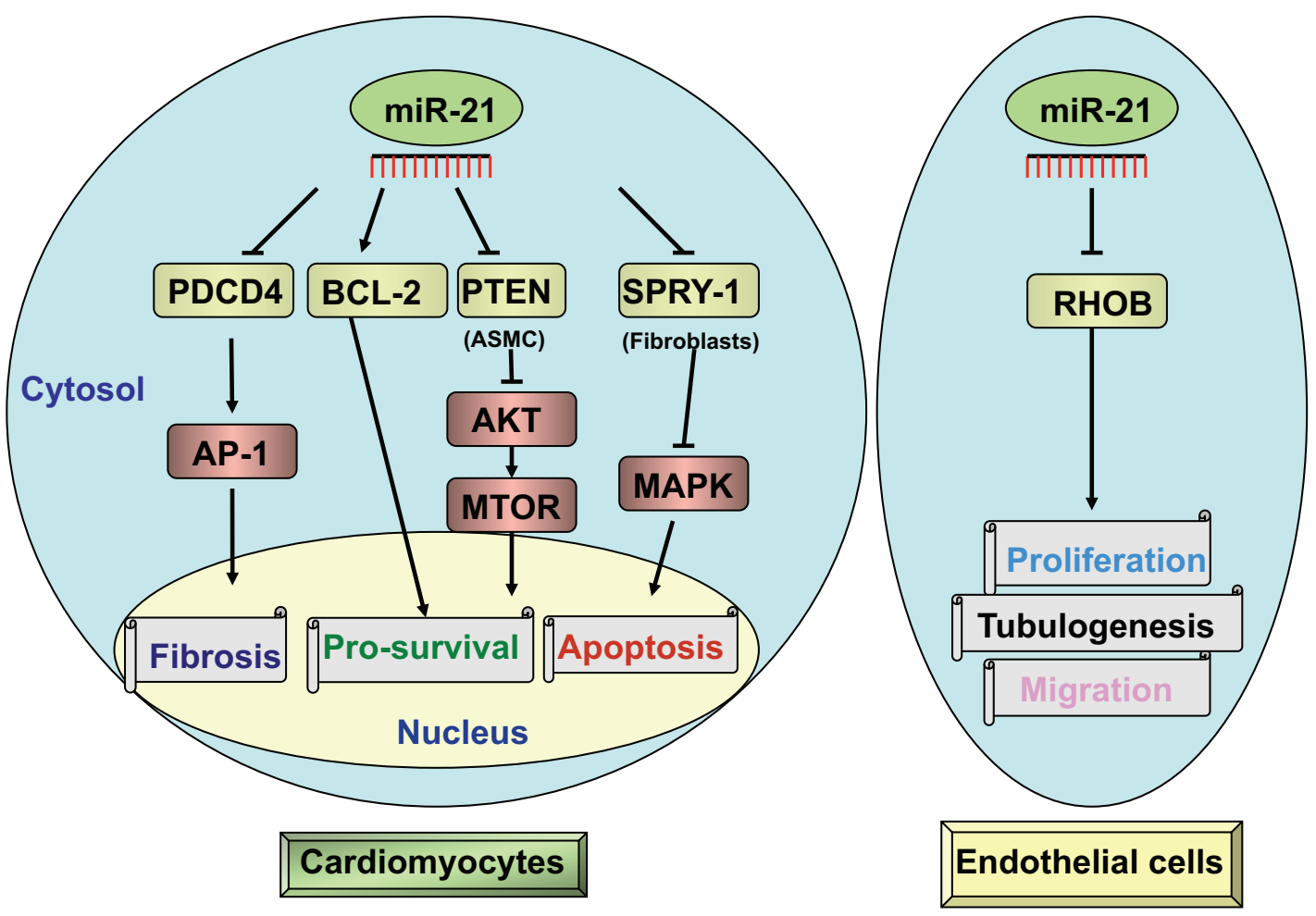

Fig. (2). Modulation of proliferation, differentiation, inflammation or apoptosis by miR-21 in smooth muscle cells or endothelial cells. miR-21 is up-regulated by TGF- $\beta$ and BMP and down-regulated in cells with serum withdrawal. The expression of miR-21 can increase the proliferation of smooth muscle cells through down-regulation of target genes, such as SP1, PTEN, SPRY1, or SPRY2 (fibroblast), or up-regulation of Bcl-2. Furthermore, miR-21 can up-regulate the differentiation of smooth muscle cells through down-regulation of PDCD4. In endothelial cells, miR induced apoptosis through down-regulation of PTEN and decreased the inflammation through targeting PPAR- $\alpha$. Arrows $(\rightarrow)$ represent up-regulation. Capped lines $(\dashv)$ indicate inhibition. Abbreviation: Aortic smooth muscle cell (ASMC), B cell leukemia/lymphoma 2 (Bcl-2), bone morphogenetic protein (BMP), cystathionine $\mathrm{y}$-lyase (CSE), Jun oncogene (AP-1), phosphatase and tensin homolog (PTEN), programmed cell death 4 (PDCD4), monocyte chemotactic protein 1 (MCP-1), oscillatory shear stress (OSS), peroxisome proliferator activated receptor- $\alpha$ (PPAR- $\alpha$ ), specificity protein-1 (SP1), sprouty homologue 1 (Spry1), transforming growth factor- $\beta$ (TGF- $\beta$ ), unidirectional shear stress (USS), vascular cell adhesion molecule (VCAM).

remodeling [38]. These studies indicated that downregulation of miR-29 had a protective effect against ischemia/reperfusion-related injury [39]. Activation of PPARr agonist-pioglitazone could effectively down regulate the expression of miR-29 and up-regulate the levels of anti-apoptotic protein to decrease the myocardial infarct area [40]. However, inhibition of miR29 by PPARr agonist had a detrimental effect in cardiac fibrosis [41]. Indeed, to determine whether miR-29 is to be up-regulated or down-regulated in cardiovascular diseases is a great challenge. The inhibition of miR-29 could protect cardiomyocytes from ischemic injury, but it could also aggravate fibrosis.

\section{miR-126}

miR-126 is abundant in endothelial cells and is located within intron 7 of the Egfl7 gene [42]. It regulates angiogenesis during the stages of embryonic development $[42,43]$. Studies have shown that miR126 deficiency is linked to leaky blood vessels and hemorrhage in mice, implying that blood vessels in the mice were under developed. The mechanisms behind these phenotypes implied that the knockdown of miR126 could increase expression of their target genes,
PIK3R2 and SPRED1. It is also implied that PIK3R2 could inhibit the PI3K-AKT signaling pathway; while SPRED1 could inhibit the ras-MEK-ERK signaling pathway. In fact, both signaling pathways, and thus angiogenesis, are inhibited resulting in injury to the blood vessels [44]. While being associated with angiogenesis, miR-126 is also linked to chemotaxis and repair in endothelial cells. It has been reported that knockdown of miR-126 increased the expression of CXCL12 in vitro, followed by induction of CD34progenitor cell migration. Down-regulation of miR-126 in the hindlimb ischemia of rats also increases the levels of CXCL12, thus increasing the numbers of Sca$1^{+}$progenitor cells required for repairing ischemic injuries [45]. Furthermore, miR-126 can inhibit inflammation via direct inhibition of vascular cell adhesion molecule-1 expression, which prevents leukocyte adhesion to endothelial cells. Apart from modulating the target genes intracellularly, miR-126 also regulates genes via paracrine signaling. The endothelial cell, while undergoing apoptosis, may release microparticles or miR-126 containing apoptotic bodies to affect recipient vascular cells. Studies have shown that in atherosclerosis, miR-126 released by 
microparticles targeted and inhibited the expression of RGS16, followed by activation of CXCR4, and finally increased expression of CXCL12 [46].

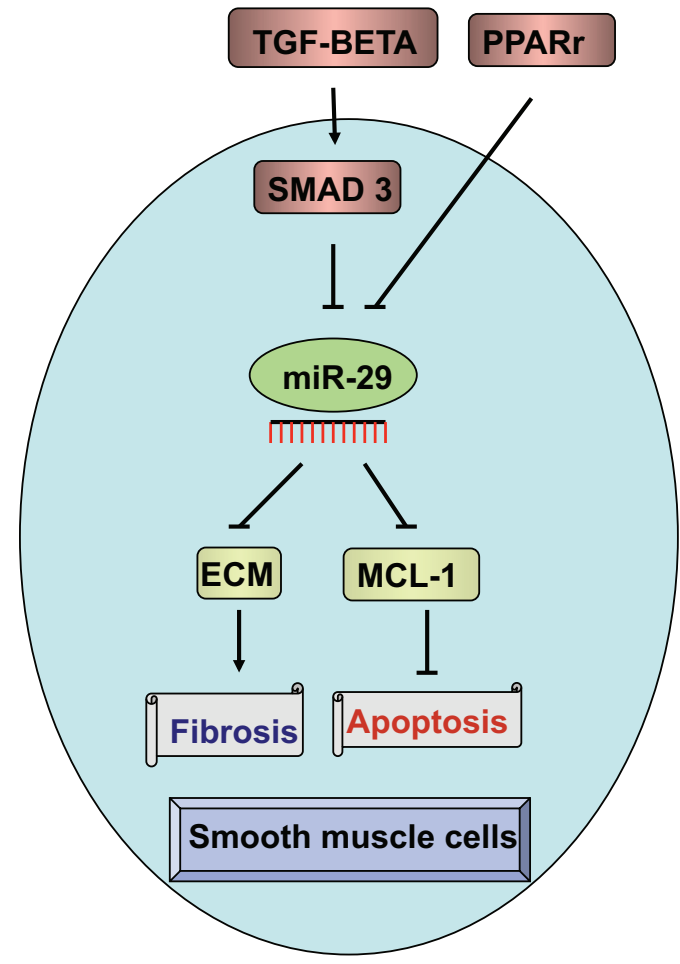

Fig. (3). Modulation of fibrosis or apoptosis by miR-29 in smooth muscle cells. Abbreviation: miR-29, induced by TGF$\beta$ - smad3 signaling, down-regulated extracellular matrix protein (ECM) synthesis to mitigate the fibrosis. Also, miR-29, suppressed by PPARy, down-regulated Mcl-1 to trigger apoptosis. Arrows $(\rightarrow)$ represent up-regulation. Capped lines $(f)$ indicate inhibition. Abbreviation: extracellular matrix (ECM), peroxisome proliferator-activated receptor Y (PPARY), transforming growth factor- $\beta$ (TGF- $\beta$ ).

Up-regulation of CXCL12 inhibited atherosclerosisinduced injuries, and CXCL12 could recruit the Sca- $1^{+}$ progenitor cells into the cell walls to repair the injury [46] (Fig. 4). In summary, miR-126 has the effect of proangiogenesis, anti-inflammation, and antiatherosclerosis. Hence, it is a promising strategy to use the microvehicle-mediated miRs in patients with atherosclerosis [47].

\section{miR-130A}

The expression of miR-130a is low in quiescent endothelial cells, but it can be activated by addition of serum to the cells. miR-130a binds to the 3'UTR of mesenchyme homeobox 2 and homeobox A5 leading to their inhibition which in turn results in enhanced angiogenesis and regulation of proliferation and migration in vascular endothelial cells [48] (Fig. 5).

\section{miR-133}

Both mir-133 and miR-1 may be transcribed into one single transcript, which can then be processed into two independent and mature miRs with individual functions [49]. Both miR-133a-1 and miR-133a-2 are expressed in cardiac smooth muscle cells and skeletal muscle cells to regulate differentiation and proliferation. However, miR-133b is not expressed in cardiac cells. Researchers have found three members of miR-133 family which correlate with other miR clusters to develop different functions, for example, miR-133a1/miR-1-2, miR-133a-2/MiR-1-1 or miR-133b/miR206 [13]. Researchers found that in patients with heart failure or hypertrophy [14], or in the animal model of TAC- or PE-induced hypertrophy, the expression of miR-133 was down-regulated. It was suggested that miR-133 has a protective effect on heart failure or hypertrophy. Adenovirus-mediated over-expression of miR-133a can down regulate PE-induced cardiac hypertrophy and decrease cardiomyocytic size [50]. Studies have shown that up-regulation of miR-133 can target the expression of calcineurin, in contrast, downregulation of miR-133 can suppress the decrease in calcineurin expression, leading to increased expression of nuclear factor in activated T cells transcription factor (NFAT), followed by induction of hypertrophy [51] (Fig. 6).

Studies have shown that RhoA and Cdc42 both played identical roles with calcineurin in hypertrophy. Furthermore, Nelf-A/WHSC2, another target gene of miR-133, was involved in the formation of cardiomyocytes [50]. Except for regulating cardiac hypertrophy, miR-133 can also prevent fibrosis in response to pressure. It has been shown that while the expression of connective tissue growth factor was increased, the expression of miR-133 was decreased in the hypertrophic tissue in response to pressure or the patients presenting with a hypertrophic heart [52]. This resulted in the accumulation of collagens and lead to the development of fibrosis of the heart. In summary, miR-133 is an important modulator of hypertrophy and has been suggested as therapeutic target for prognosis or diagnosis [50].

\section{miR-143/145}

Both miR-143 and miR-145 were transcribed bicistronically and both are expressed in the cardiomyocytes during cardiac development. They are also expressed in the smooth muscle cells of the adults [53]. The biogenesis of miR-143/145 was transcribed by the transcription factor, serum response factor (SRF), which binds to the CArG box of DNA with two cofactors, myocardin and myocardin-related transcription factors. The synthesis of miR-143/145 sent cells into contractile or differential state $[54,55]$. Furthermore, TGF- $\beta$ or BMP4 induced expression of miR143/miR145 via cofactor, myocardin or myocardinrelated transcription factors, respectively [56] (Fig. 7). Moreover, some researchers found that Jag-1 could activate notch intracellular domain, translocating into the nucleus, where it binds to CBF1 [57]. Both of them cooperatively activated $\mathrm{miR}-143 / \mathrm{miR}-145$ to differentiate cells into a state of contraction, and this process was independent of SRF or CArG box [57]. 


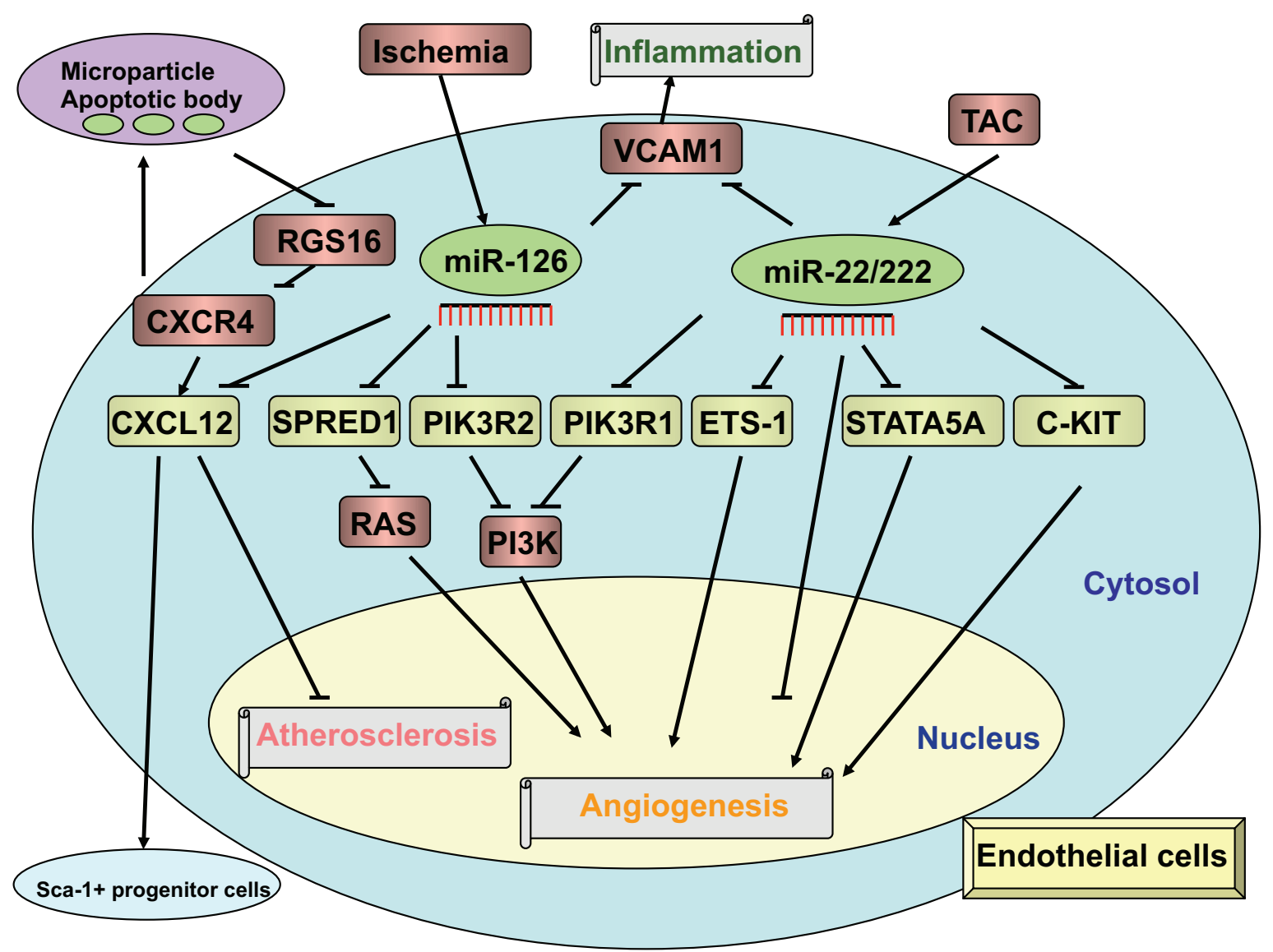

Fig. (4). Modulation of fibrosis or apoptosis by crosstalk of miR-126 and miR-221/222 in endothelial cells. The biogenesis of miR-126 is synthesized by the two transcription factors, ETS-1 and ETS-2 and cofactor, KLF2. miR-126 enhances angiogenesis through the de-regulation of PIK3R2 (the inhibitor of PI3K signaling) or SPRED1 (the inhibitor of ras-raf-MEK-ERK signaling). However, miR-126 enhances angiogenesis through the down-regulation of PIK3R1. Furthermore, miR-126 can inhibit inflammation via targeting VCAM1, which is also down-regulated by miR-221/222. miR-126 can act in an anti-atherosclerosic manner for the release of microparticle containing miR-126 and inhibition of the expression of RGS-16. The RGS-16 downregulated CXCR4 to increase the expression of CXCL12 and was also down-regulated by miR-126. miR-126 inhibits atherosclerosis growth and recruited progenitor cells to repair the endothelial cells. In addition, miR-221/222 promoted the angiogenesis by targeting STATA5A. Arrows $(\rightarrow)$ represent up-regulation. Capped lines $(-1)$ indicate inhibition. Abbreviation: chemokine (C-X-C motif) ligand 12 (CXCL12), chemokine (C-X-C motif) receptor 4 (CXCR4), cyclin-dependent kinase inhibitor 1B (p27/Kip1), cyclin-dependent kinase inhibitor 1C (p57/Kip2), E26 transformation-specific sequence-1 (ETS-1), E26 transformation-specific sequence-2 (ETS-2), phosphoinositol-3 kinase (PI3K) regulatory subunit 2 (PIK3R2), regulator of Gprotein signaling 16 (RGS16), signal transducer and activator of transcription 5A (STATA5A), sprouty-related protein EVH1 domain containing 1(SPRED1), vascular cell adhesion molecule 1 (VCAM-1).

The phenotypes of smooth muscle cells were modulated by miR-143 and miR-145. In quiescent cells, the phenotype is in the contractile or differentiation state; however, cells exposed to stress switched from this state to the proliferative or synthetic state. Upon synthesis of $\mathrm{miR}-143 / \mathrm{miR}-145$, they could regulate the same genes cooperatively, and down-regulated the targets, klf4 and klf5, to increase the differentiation of cells and expression of their markers, SMA, calponin, and SM22- $\alpha$. Transformation of the phenotype from contractile state to the proliferation state is indicative of vascular remodeling. For example, knockdown of miR$143 / \mathrm{miR}-145$ can down-regulate the myocardin expression and lead to transformation of the contractile phenotype to the proliferative phenotype [56]. In gain of function experiments, over-expression of miR-145 can suppress the formation of neo-intimal layer, and increase the marker of smooth muscle cells to approach the contractile phenotype [58]. Up-regulation of miR-143/miR-145 mitigated TAC or carotid artery occlusion induced neo-intimal formation. In contrast, loss of function of miR-143/145 in miR-143/miR-145 knockout mice resulted in neo-intimal formation. Neointimal formation is regulated by miR-143/miR-145. It has been shown that miR-145 can target the ETS-like gene 1 (Elk-1) to inhibit the proliferation of smooth muscle cells [53]. Furthermore, angiotensin Iconverting enzyme levels were increased in miR$143 / 145$ knockout mice, which had a higher occurrence of atherosclerosis lesions compared to wild type mice. Besides Elk-1 and angiotensin l-converting enzyme, calmodulin kinase II was also found to be involved in the modulation of calcium in apoptosis. 


\section{Serum}

\section{Proangiogenic factors}

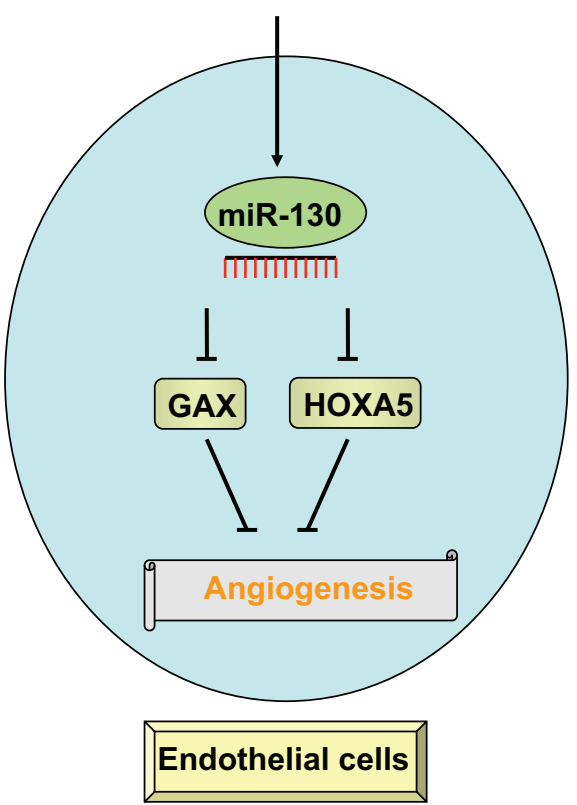

Fig. (5). Modulation of angiogenesis by miR-130 in endothelial cells. miR-130a induced by serum or proangiogenic factors binds the 3'UTR of the mesenchyme homeobox 2 (GAX) and homeobox A5 (HoxA5) to inhibit them, thus enhancing angiogenesis in endothelial cells. Arrows $(\rightarrow)$ represent up-regulation. Capped lines $(\dashv)$ indicate inhibition. Abbreviation: proteins mesenchyme homeobox 2 (GAX), Homeobox A5 (HoxA5).

\section{miR-155}

miR-155 is expressed in smooth muscle cells, endothelial cells, and macrophages. As inflammation develops, these three cell-types work interactively with each other. For example, oxidized LDL, or shear stress, induced the activation of endothelial cells, which in turn recruited monocytes; the precursor cells of macrophages, to the cell wall. Following recruitment the monocytes then differentiate into macrophages, which infiltrate the smooth muscle cell layer inducing proliferation in these cells [59].

When macrophages activated and induced inflammatory response, the expression of miR-155 was increased. Some molecules could either up-regulate or down-regulate the expression of miR-155. For example, Toll-like receptor ligands can up regulate the synthesis of miR-155 in macrophages or monocytes. However, IL-10, an anti-inflammatory cytokine, downregulates the expression of miR-155 through inhibition of STAT3. This in turn increased the activity of SH2containing inositol polyphosphate-5-phosphatase and suppressed the production of pro-inflammatory cytokines, subduing the inflammatory response [60] (Fig. 8). Many target genes of miR-155 have now been discovered. For example, up-regulation of miR-155 can inhibit TGF-beta-induced phosphorylation of SMAD2, which in turn up-regulates the production of IL-1 $\beta$ and aggravates the inflammatory response [61]. Furthermore, miR-155 also binds the 3'UTR of suppressor of cytokine signaling 1 [62], or B-cell leukemia/lymphoma 6 [63] which further aggravate the inflammatory response [64]. In contrast, it also down-

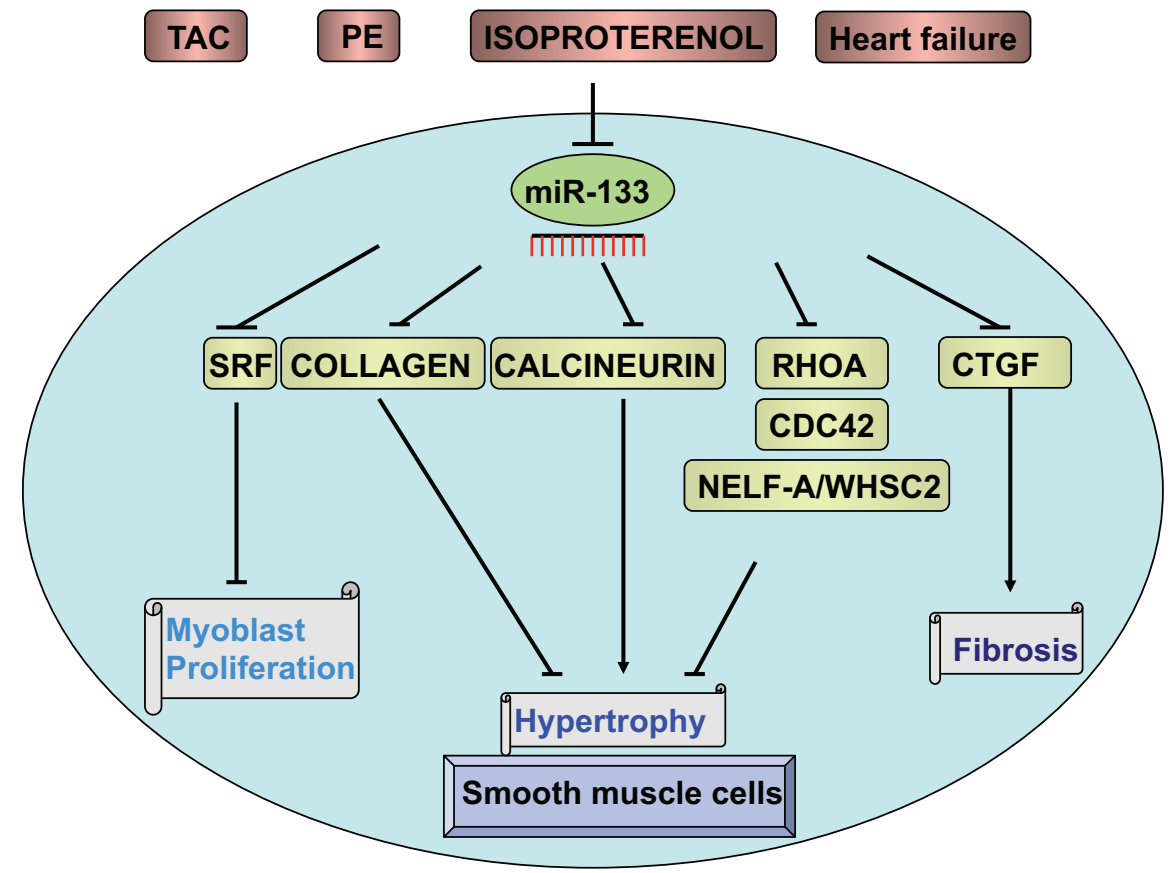

Fig. (6). Modulation of the hypertrophy or fibrosis of miR-130 in smooth muscle cells. miR-133 enhanced the hypertrophy through inhibition of RhoA, Cdc42, and Nelf-A/WHSC2. In addition, miR-133 enhanced the proliferation of myoblasts via inhibition of SRF. However, miR-133 suppressed hypertrophy via inhibition of calcineurin. Furthermore, fibrosis progression was increased by miR-133 via inhibition of CTGF. Arrows $(\rightarrow)$ represent up-regulation. Capped lines $(\dashv)$ indicate inhibition. Abbreviation: Cdc42 (Rho-type GTPase), connective tissue growth factor (CTGF), serum response factor (SRF). 


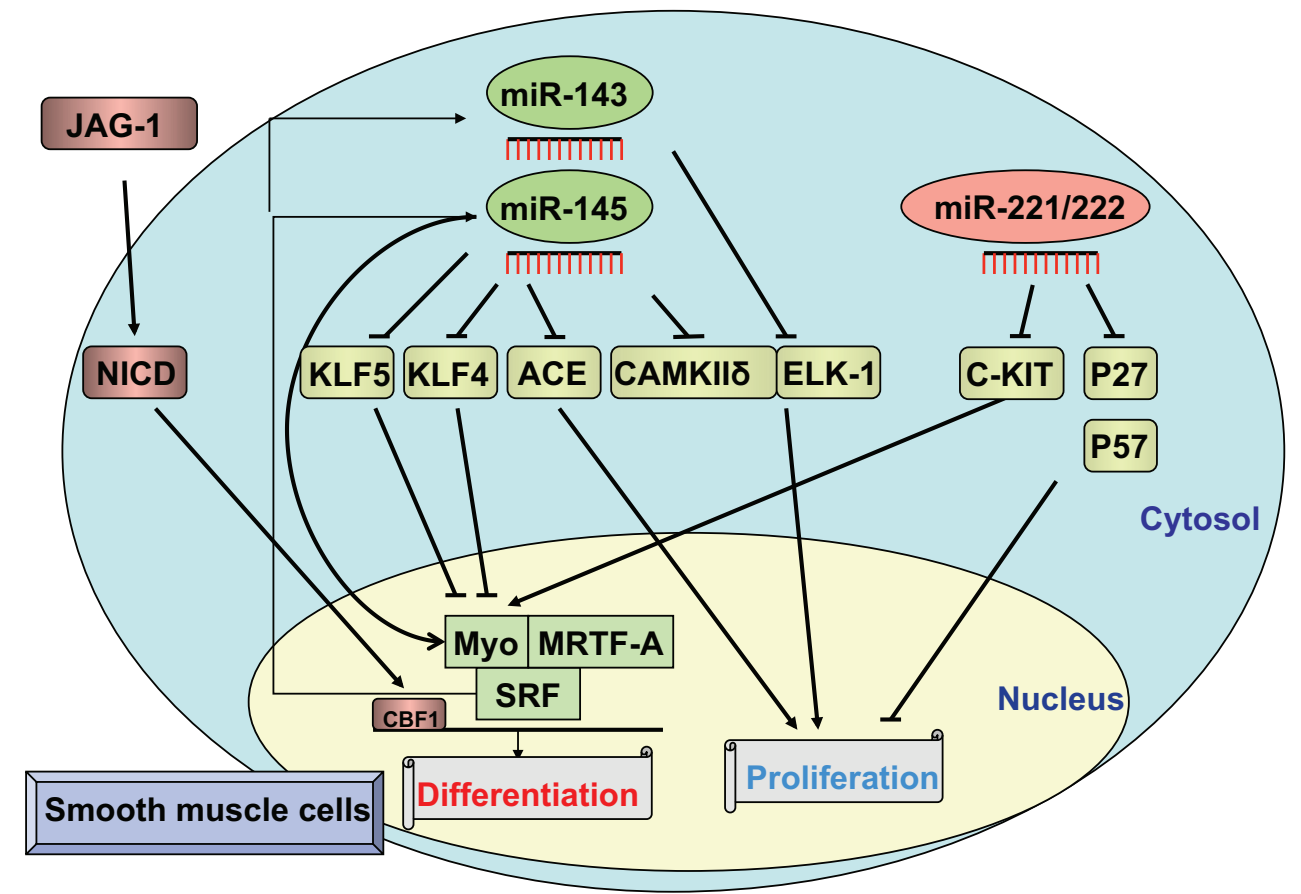

Fig. (7). Modulation of differentiation and proliferation by crosstalk of miR-143/145 and miR-221/222 in smooth muscle cells. The biogenesis of miR-143/145 is synthesized by the transcription factor, SRF and two cofactors, myocardin (Myo) and MRTFA, which lead to differentiation. The synthesis of miR-143/145 was increased by Jag-1-induced activation of NICD, in cooperation with CBF1. The expression of miR-143/145 is inhibited by miR-221/222 through inhibition of myocardin via C-kit. miR-145 also induced the expression of myocardin through inhibition of KLF4 and KLF5 to stimulate differentiation. In addition, miR-145 suppresses proliferation via inhibition of Elk-1 and ACE. Furthermore, miR-145 targets the calmodulin kinase II $\delta$ (Camkllס) to suppress apoptosis. miR-221/222 also binds to the 3'UTR of p27 or p57 to inhibit proliferation. Arrows ( $\rightarrow$ ) represent up-regulation. Capped lines $(-)$ indicate inhibition. Abbreviation: angiotensin l-converting enzyme (ACE), calmodulin kinase II $\delta$ (Camkll $\delta)$, cyclin-dependent kinase inhibitor 1C (c-Kit), cyclin-dependent kinase inhibitor 1B (p27), cyclin-dependent kinase inhibitor 1C (p57), ETS-like gene 1 (Elk-1), Krüppel-like factor 4 (KLF 4), Krüppel-like factor 5 (KLF 5), myocardin (Myo), myocardin-related transcription factor (MRTF), serum response factor (SRF).

regulated the expression of TGF- $\beta$ activated kinase $1 /$ MAP3K7 binding protein 2 [58], suppressing the inflammatory response. In endothelial cells, miR-155 played a negative role in inflammation. Studies have shown that unidirectional shear stress can up-regulate the synthesis of miR-155, which targets two genes, angiotensin-II type-1 receptor or Ets-1 [65], suppressing inflammation. In cardiomyocytes, miR-155 increased hypertrophy via down-regulation of MEF2A, Jarid 2, or Jumonji [14]. In summary, miR-155 inhibits hypertrophy in cardiomyocytes and plays an inhibitory role in endothelial cells, yet in contrast activates the inflammatory response.

\section{miR-204}

miR-204 is located in the intron 6 of transient receptor potential melastatin 3 gene in chromosome 9 [66]. Studies have shown that miR-204 was downregulated in a rat model of pulmonary artery hypertension, simulated by exposing the rats to chronic hypoxia or monocrotaline, implying miR-204 could play a protective in pulmonary artery hypertension [67]. Pulmonary artery hypertension has several phenotypes, such as the proliferation of smooth muscle cells, the resistance to apoptosis, or induction of muscle contraction. Firstly, studies have reported that
miR-204 plays a role in proliferation through the SrcSTAT3-NFAT signaling pathway.

They also reported that suppression of miR-204 was induced by either endothelin-1 or PDGF via activation of STAT3. Down-regulation of miR-204 increased the activity of SHP2, which in turn led to the activation of src, followed by activation of STAT3 [68] (Fig. 9). Studies reported that STAT3 can activate Pim1 to up-regulate NFATc2 [69]. NFATc2 decreased the expression of the voltage-gated potassium channels, $\mathrm{Kv} 1.5$, followed by depolarization which opened the calcium channel. Intracellular concentrations of calcium and potassium were increased by the opened channels, inducing the proliferation of smooth muscle cells [70]. Furthermore, NFAT can also increase the expression of $\mathrm{Bcl}-2$, an anti-apoptosis protein [71]. It has been implied that miR-204 may suppress the proliferation of smooth muscle cells and increase apoptosis via inhibition of NFAT [65]. miR-204 or NFAT may represent potential therapeutic targets to treat pulmonary artery hypertension. Besides the NFAT signaling pathway, miR-204 also regulates the BMPR2 signaling pathway via IL-6 [72]. The expression of IL-6 is increased in cases of pulmonary artery hypertension. Over-expression of IL- 6 could stimulate the progression of this disease [72]. IL-6 can activate STAT3 to up- 


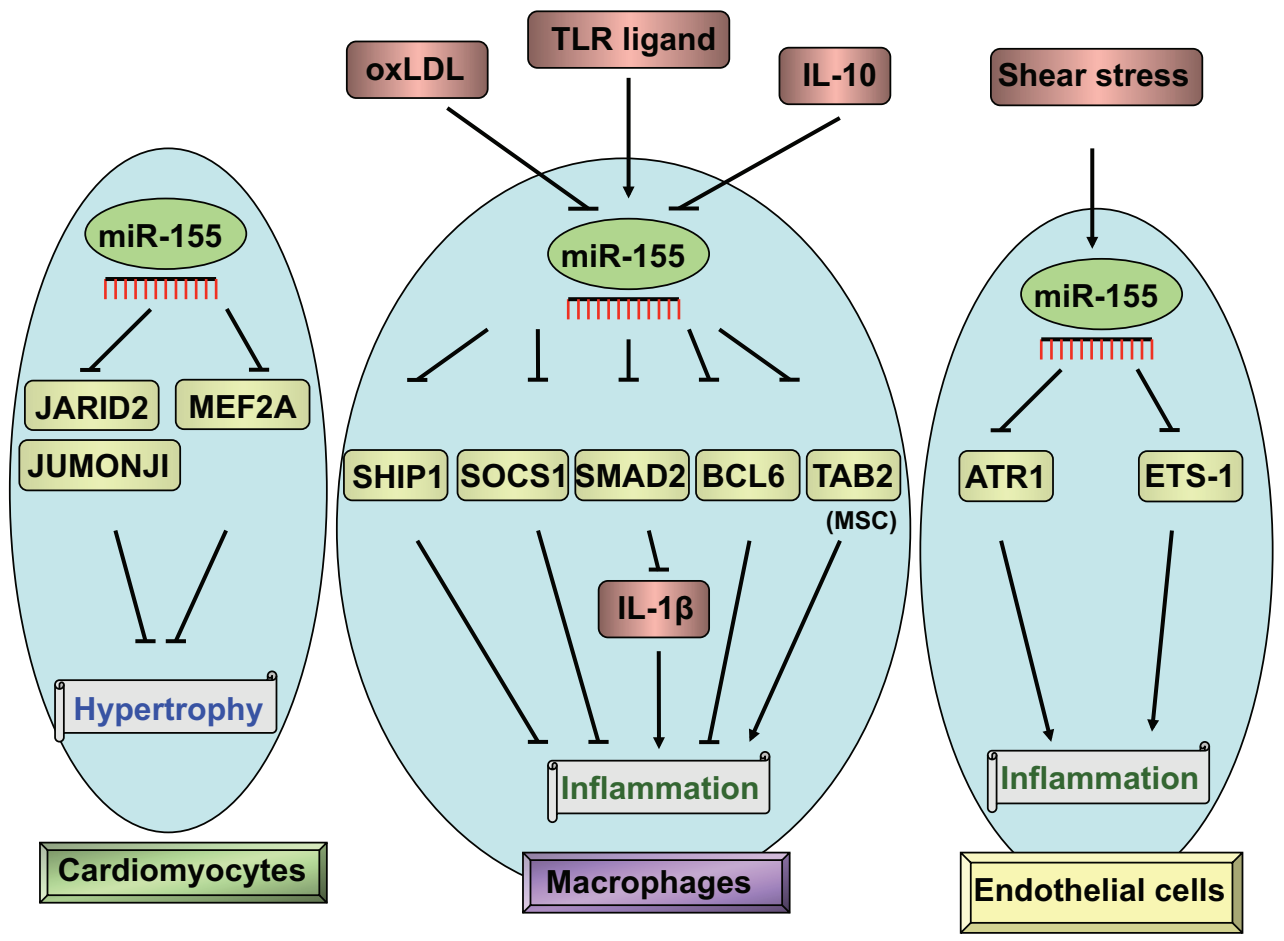

Fig. (8). Modulation of inflammation by miR-155 in macrophages or endothelial cells. The expression of miR-155 is activated by TLR ligand, oxidized LDL (oxLDL)/interferon-y (IFN-y), or inhibited by lipopolysaccharide, oxidized LDL and interleukin-10. miR155 inhibited inflammation by inhibition of SHIP1, SOCS1, SMAD2 or Table 2 (mesenchymal cells) or activated inflammation by targeting the Bcl-6 in macrophages. miR-155, induced by shear stress, inhibited inflammation in endothelial cells via downregulation of ATR1 and ETS-1. Arrows $(\rightarrow)$ represent upregulation. Capped lines $(-1)$ indicate inhibition. Abbreviation: angiotensin II type-1 receptor (AT1R), B-cell leukemia/lymphoma 6 (Bcl6), E26 transformation-specific sequence-1 (ETS-1), interferon- $\gamma$ (IFN- $\gamma$ ), interleukin-10 (IL-10), inositol polyphosphate-5-phosphatase (SHIP1), lipopolysaccharide (LPS), MAD homolog 2 (SMAD2), oxidized LDL (oxLDL), suppressor of cytokine signaling 1 (SOCS1), TGF- $\beta$ activated kinase 1/MAP3K7 binding protein 2 (Table 2), Toll-like receptor (TLR) ligands.

regulate the expression of miR-17/92. miR-17/92 can bind the 3'UTR of BMRP to inhibit the proliferation of smooth muscle cells. miR-204 and miR-17/92 modulate this disease with the same molecules.

\section{miR-221/miR-222}

miR-221 and miR-222 are transcribed from a gene cluster in chromosome $\mathrm{X}$, and have identical seed sequences. They are abundantly expressed in both smooth muscle cells and endothelial cells [73]. Both miR-221 and miR-222 can up-regulate proliferation of smooth muscle cells in order to re-grow the intimal formation via two mechanisms. One involves the induction of proliferation of smooth muscle cells, and the other involves inhibition of the phenotype to switch from a contractile state to a proliferative state. Regarding up-regulation of proliferation, some studies have shown that miR-221 is related to hypertrophy. In animal models of TAC-induced hypertrophy and patients presenting with the disease, miR-221 was significantly up-regulated. Indeed, cardiomyocytes transferred with mimics of miR-221 induced hypertrophy in these cells and, in contrast, downregulation of miR-221 will reverse this phenotype [74]. Furthermore, adenovirus mediated over-expression of miR-221/222 increased the neointimal growth. However, knockdown of miR-221/222 with antogomirs decreases its growth [75]. Some target genes of miR$221 / 222$ have been outlined. Up-regulation of miR$221 / 222$ by balloon angioplasty injury of coronary artery decreased the two target genes, p27 (Kip1) and p57 (Kip2), and increased the intimal injury and proliferation of smooth cells $[75,76]$. While miR-221/222 also downregulated the contractile state resulting in injury to the heart. It has been reported that miR-221/222 can down-regulate the expression of c-kit [77, 78] (Fig. 7). This in turn inhibited the synthesis of myocardin, the synthetic cofactor of miR-143/145, resulting in inhibition of the synthesis of mi-143/145, which is related to the contractile state. In endothelial cells, miR-221/222 displayed anti-angiogenetic, atheroprotective and antiinflammatory properties. Firstly, studies in which cells were exposed to miR-221/222 showed down-regulation in the expression of ETS-1, the transcription factor required for synthesis of $\mathrm{miR}-126$, related to angiogenesis [65] (Fig. 4). Furthermore, miR-221/222 also targeted C-kit or anti-PI3K signaling molecule, PIk3R1, and led to down-regulation of angiogenesis. Secondly, up-regulation of miR-221/222 induced by inflammatory molecules or basic fibroblastic growth factor down-regulated its target gene, signal transducer and activator of transcription $5 \mathrm{~A}$, related with formation of atherosclerosis. The production of miR-222 could be considered a protective mechanism in the blood vessel [25]. Thirdly, miR-221/222 is involved in 


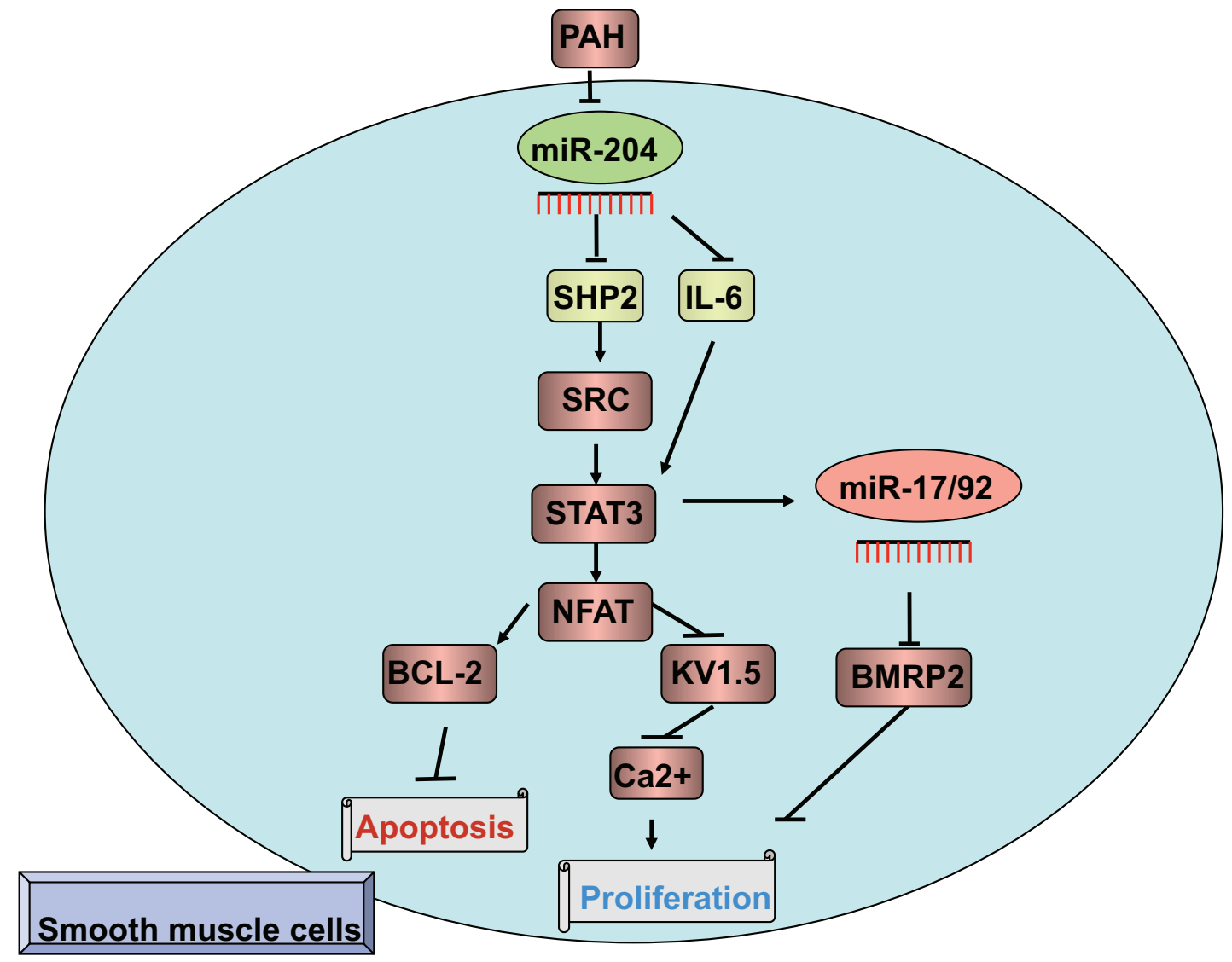

Fig. (9). Modulation of proliferation by crosstalk of miR-204 and miR-17/92 in smooth muscle cells. miR-204 stimulated proliferation through the Src-STAT3-NFAT signaling pathway by targeting SHP2. NFAT decreased the expression of the voltage-gated potassium channels, Kv1.5, to inhibit proliferation. Besides the NFAT signaling pathway, miR-204 also increased the BMPR2 signaling pathway through inhibition of IL-6, followed by down-regulation of STAT3. Arrows ( $\rightarrow$ ) represent upregulation. Capped lines $(\dashv)$ indicate inhibition. Abbreviation: interleukin-6 (IL-6), nuclear factor of activated T-cells (NFAT), Pulmonary artery hypertension (PAH), signal transducer and activator of transcription 3 (STAT3).

inflammation. miR-221/222 mitigated inflammation through inhibition of the target gene, vascular cell adhesion molecule [65]. In summary, miR-221/222 aggravated the intimal growth in SMCs, but mitigated the angiogenesis, inflammation or atherosclerosis in ECs.

\section{miR-378}

miR-378 is expressed in cardiomyocytes, but not in cardiac fibroblasts. The expression of miR-378 was down-regulated significantly in cases of cardiac hypertrophy and heart failure [79], implying that miR378 could be an inhibitor of these diseases. Overexpression of miR-378 can inhibit the epinephrineinduced cardiac hypertrophy through the downregulation of growth factor receptor-bound protein2, and inhibit the raf-MEK-ERK or PI3K-AKT signaling pathway through ras [79] (Fig. 10). miR-378 can inhibit ras-related signaling pathway to prevent from cardiac hypertrophy, and in other words, the deficiency of miR378 may promote the occurrence of hypertrophy. It has been shown that miR-378 can down-regulate the expression of IGF1R to up-regulate apoptosis [80]. In other words, knockdown of miR-378 with antagomir may protect cardiomyocytes from $\mathrm{H}_{2} \mathrm{O}_{2}$-induced and $\mathrm{H} / \mathrm{R}$-induced injury through up-regulation of IGF1R [81]. Therefore, miR-378 is involved in cardiac remodeling and the survival of cardiomyocytes.

Owing to the fact that these miRs are differentially expressed in cardiovascular diseases, some scientists attempted to clarify miRs profiling with in vitro or in vivo animal models. And in patients with cardiovascular diseases, miRs profiling could be served as a biomarker for future diagnosis or prognosis tools. At present, manipulation of these miRs could be another effective strategy for anti- hypertrophy, anti-fibrosis, and anti-angiogenesis treatment. There are two common methods to manipulate target genes related with cardiovascular diseases through miRs. The first utilizes binding of miR mimic, which is synthesized by double stranded RNA, to its target genes and disable function of target gene. The other method exploits binding of antagomir, manufactured by the single stranded oligonucleotide, to its $\mathrm{miR}$, down-regulates $\mathrm{miR}$ and strengthens its target genes' expression. Many preclinical and clinical trials of miRs have been proposed and carried out in the fields of cancer and cardiovascular diseases. In cancer research, miRs are 


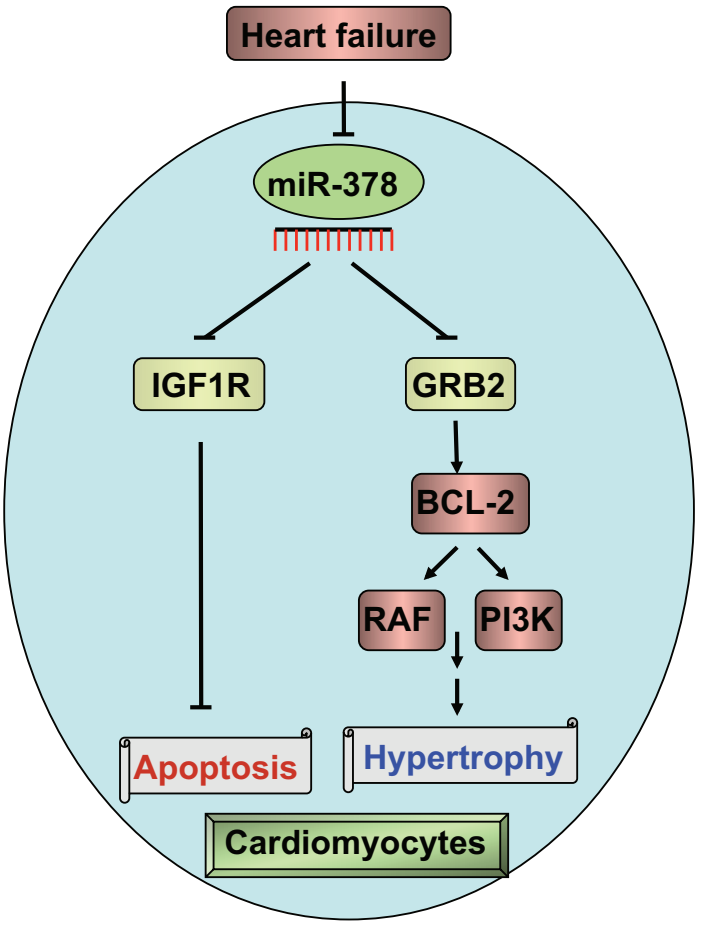

Fig. (10). Modulation of proliferation and apoptosis by miR378 in cardiomyocytes. Up-regulation of miR-378 increased apoptosis via inhibition of IGF1R related signaling. Overexpression of miR-378 could inhibit the epinephrine-induced cardiac hypertrophy through the down-regulation of growth factor receptor-bound protein2 (Grb2), followed by inhibition of Ras to inhibit the raf-MEK-ERK or PI3K-AKT signaling pathway. Arrows $(\rightarrow)$ represent up-regulation. Capped lines $(f)$ indicate inhibition. Abbreviation: growth factor receptorbound protein2 (Grb2), insulin-like growth factor 1 (IGF1).

classified into oncogenic miRs, for example, miR-10b, mir17-92, miR-21or miR-155 and tumor suppressor miRNA, let-7, miR-34, miR-26a, miR-126 and miR-155, etc. miR-34 and let-7 have entered into clinical trial for evaluating the therapeutic effect on cancer patients. In the field of cardiovascular diseases, mir-208 and mir195 are two candidate miRs for future application in human beings. miR-208 KO mice compared to wildtype littermates have few phenotypes of cardiac hypertrophy and fibrosis [82]. mir-208 is suitable for developing its antagomir to treat diseases related with heart failure. miR-195 expression is elevated in cardiac hypertrophy animal model, and its over-expression induces heart failure in transgenic mice [83]. miR-122 antagomir, miravirsen, is quickly developed for the treatment of hepatitis $\mathrm{C}$ in clinical trial because of its inhibitory function of viral replication. Mir-122 could inhibit the HCV RNA in serum [84]. With no side effect of miRs in cancer therapy, miRs are expressed in normal cells and not in cancer cells. The problems of preservation and delivery to the target site can be improved by increasing stability of the treatment of diseases with miRs from chemical modification in the synthesis of miRNA drug. Progression of miRs delivery has been improved according to the delivery method of siRNA by liposome and polymer.

\section{CONCLUSION}

A growing body of evidence suggests that miRs play crucial roles in vascular remodeling. For example, miRs can modulate the proliferation or differentiation state of phenotype in smooth muscle cells, the proliferation or repair of endothelial cells, and the inflammatory or anti-inflammatory response of macrophages. There are still many challenges to find adequate strategies to modulate these miRs, the targets of these miRs and the phenotypes related to them. Anti-miRs based therapy has developed, for example; loss of function of miRs with antagomirs or locked nucleic acid-based oligonucleotide, gain of function of miRs with mimics, or virus-mediated transfer of miRs have been developed to treat the cardiovascular diseases related to vascular remodeling. The problems of dosage, specificity, mode of delivery, efficiency, or the half-lives of these molecules must be considered.

\section{CONFLICT OF INTEREST}

The authors confirm that this article content has no conflict of interest.

\section{ACKNOWLEDGEMENTS}

YCH was supported by grants NSC 101-2314-B182A-137-MY3 from the National Science Council, Republic of China, and by grants CMRPG 2A0203 and CMRPG 2A0213 from Chang Gung Memorial Hospital, Taiwan, ROC. FYC was supported by grants CMRPG 2D0021 from Chang Gung Memorial Hospital, Taiwan, ROC.

\section{REFERENCES}

[1] Makanya AN, Hlushchuk R, Djonov VG. Intussusceptive angiogenesis and its role in vascular morphogenesis, patterning, and remodeling. Angiogenesis 2009; 12: 113-23.

[2] Raffetto JD, Khalil RA. Matrix metalloproteinases and their inhibitors in vascular remodeling and vascular disease. Biochem Pharmacol 2008; 75: 346-59.

[3] Grant JS, White K, MacLean MR, Baker AH. MicroRNAs in pulmonary arterial remodeling. Cell Mol Life Sci 2013; 70 : 4479-94.

[4] Chi Z, Melendez AJ. Role of cell adhesion molecules and immune-cell migration in the initiation onset and development of atherosclerosis. Cell Adh Migr 2007; 1: 171-5.

[5] Klein LW. Atherosclerosis regression vascular remodeling and plaque stabilization. J Am Coll Cardiol 2007; 49: 271-3.

[6] Chen NX, Kiattisunthorn K, O'Neill KD, et al. Decreased microRNA is involved in the vascular remodeling abnormalities in chronic kidney disease (CKD). PLoS One 2013; 8: e64558.

[7] Morrell NW, Adnot S, Archer SL, et al. Cellular and molecular basis of pulmonary arterial hypertension. J Am Coll Cardiol 2009; 54: S20-31.

[8] Kang SS, Gosselin C, Ren D, Greisler HP. Selective stimulation of endothelial cell proliferation with inhibition of smooth muscle cell proliferation by fibroblast growth factor-1 plus heparin delivered from fibrin glue suspensions. Surgery 1995; 118: 280-6.

[9] Sales VL, Engelmayr GC Jr, Mettler BA, Johnson JA Jr, Sacks MS, Mayer JE Jr. Transforming growth factor-beta1 modulates extracellular matrix production proliferation and apoptosis of endothelial progenitor cells in tissue-engineering scaffolds. Circulation 2006; 114: I193-9. 
[10] Kim HJ, Kim MY, Jin $\mathrm{H}$, et al. Peroxisome proliferatoractivated receptor $\delta$ regulates extracellular matrix and apoptosis of vascular smooth muscle cells through the activation of transforming growth factor- $\beta 1 / \mathrm{Smad} 3$. Circ Res 2009; 105: 16-24.

[11] Kim HR, Gallant C, Leavis PC, Gunst SJ, Morgan KG. Cytoskeletal remodeling in differentiated vascular smooth muscle is actin isoform dependent and stimulus dependent. Am J Physiol Cell Physiol 2008; 295: C768-78.

[12] Latronico MV, Condorelli G. MicroRNAs and cardiac pathology. Nat Rev Cardiol 2009; 6: 419-29.

[13] Townley-Tilson WH, Callis TE, Wang D. MicroRNAs 1133 and 206: critical factors of skeletal and cardiac muscle development function and disease. Int $\mathrm{J}$ Biochem Cell Biol 2010; 42: 1252-5.

[14] Sayed D, Hong C, Chen IY, Lypowy J, Abdellatif M. MicroRNAs play an essential role in the development of cardiac hypertrophy. Circ Res 2007; 100: 416-24.

[15] Li Q, Song XW, Zou J, et al. Attenuation of microRNA-1 derepresses the cytoskeleton regulatory protein twinfilin-1 to provoke cardiac hypertrophy. J Cell Sci 2010; 123: 2444-52.

[16] Bostjancic E, Zidar N, Stajner D, Glavac D. MicroRNA miR-1 is up-regulated in remote myocardium in patients with myocardial infarction. Folia Biol (Praha) 2010; 56: 27-31.

[17] Ai J, Zhang R, Gao X, et al. Overexpression of microRNA-1 impairs cardiac contractile function by damaging sarcomere assembly. Cardiovasc Res 2012; 95: 385-93.

[18] Zhao Y, Samal E, Srivastava D. Serum response factor regulates a muscle-specific microRNA that targets Hand2 during cardiogenesis. Nature 2005; 436: 214-20.

[19] Ikeda S, He A, Kong SW, et al. MicroRNA-1 negatively regulates expression of the hypertrophy-associated calmodulin and Mef2a genes. Mol Cell Biol 2009; 29: 2193204.

[20] Elia L, Contu R, Quintavalle M, et al. Reciprocal regulation of microRNA-1 and insulin-like growth factor-1 signal transduction cascade in cardiac and skeletal muscle in physiological and pathological conditions. Circulation 2009; 120: 2377-85.

[21] Jiang $Y$, Yin $H$, Zheng XL. MicroRNA-1 inhibits myocardininduced contractility of human vascular smooth muscle cells. J Cell Physiol 2010; 225: 506-11.

[22] Curcio A, Torella D, laconetti C, et al. MicroRNA-1 downregulation increases connexin 43 displacement and induces ventricular tachyarrhythmias in rodent hypertrophic hearts. PLoS One 2013; 8: e70158.

[23] Adam O, Lohfelm B, Thum T, et al. Role of miR-21 in the pathogenesis of atrial fibrosis. Basic Res Cardiol 2012; 107: 278.

[24] Cheng $Y$, Liu X, Zhang S, Lin Y, Yang J, Zhang C. MicroRNA-21 protects against the $\mathrm{H} 2 \mathrm{O} 2$-induced injury on cardiac myocytes via its target gene PDCD4. J Mol Cell Cardiol 2009; 47: 5-14.

[25] Dentelli P, Rosso A, Orso F, Olgasi C, Taverna D, Brizzi MF. microRNA-222 controls neovascularization by regulating signal transducer and activator of transcription $5 \mathrm{~A}$ expression. Arterioscler Thromb Vasc Biol 2010; 30: 1562-8.

[26] Egito ES, Aiello VD, Bosisio IB, et al. Vascular remodeling process in reversibility of pulmonary arterial hypertension secondary to congenital heart disease. Pathol Res Pract 2003; 199: 521-32.

[27] Cardin S, Guasch E, Luo X, et al. Role for MicroRNA-21 in atrial profibrillatory fibrotic remodeling associated with experimental postinfarction heart failure. Circ Arrhythm Electrophysiol 2012; 5: 1027-35.

[28] Thum T, Gross C, Fiedler J, et al. MicroRNA-21 contributes to myocardial disease by stimulating MAP kinase signalling in fibroblasts. Nature 2008; 456: 980-4.

[29] Lin Y, Liu X, Cheng Y, Yang J, Huo Y, Zhang C. Involvement of MicroRNAs in hydrogen peroxide-mediated gene regulation and cellular injury response in vascular smooth muscle cells. J Biol Chem 2009; 284: 7903-13.

[30] Maegdefessel L, Azuma J, Toh R, et al. MicroRNA-21 blocks abdominal aortic aneurysm development and nicotineaugmented expansion. Sci Transl Med 2012; 4: 122ra122.
[31] Cheng Y, Zhang C. MicroRNA-21 in cardiovascular disease. J Cardiovasc Transl Res 2010; 3: 251-5.

[32] Patrick DM, Montgomery RL, Qi X, et al. Stress-dependent cardiac remodeling occurs in the absence of microRNA-21 in mice. J Clin Invest 2010; 120: 3912-6.

[33] Zhang Y, Huang XR, Wei LH, Chung AC, Yu CM, Lan HY. miR-29b as a therapeutic agent for angiotensin II-induced cardiac fibrosis by targeting TGF-beta/Smad3 signaling. Mol Ther 2014; 22: 974-85.

[34] Sabatel C, Malvaux L, Bovy N, et al. MicroRNA-21 exhibits antiangiogenic function by targeting RhoB expression in endothelial cells. PLoS One 2011; 6: e16979.

[35] Kriegel AJ, Liu Y, Fang Y, Ding X, Liang M. The miR-29 family: genomics cell biology and relevance to renal and cardiovascular injury. Physiol Genomics 2012; 44: 237-44.

[36] van Rooij E, Sutherland LB, Thatcher JE, et al. Dysregulation of microRNAs after myocardial infarction reveals a role of miR-29 in cardiac fibrosis. Proc Natl Acad Sci U S A 2008; 105: 13027-32.

[37] Abonnenc M, Nabeebaccus AA, Mayr U, et al. Extracellular matrix secretion by cardiac fibroblasts: role of microRNA-29b and microRNA-30c. Circ Res 2013; 113: 1138-47.

[38] Mott JL, Kobayashi S, Bronk SF, Gores GJ. mir-29 regulates $\mathrm{Mcl}-1$ protein expression and apoptosis. Oncogene 2007; 26: 6133-40.

[39] Ye Y, Perez-Polo JR, Qian J, Birnbaum Y. The role of microRNA in modulating myocardial ischemia-reperfusion injury. Physiol Genomics 2010; 43: 534-42.

[40] Ye Y, Hu Z, Lin Y, Zhang C, Perez-Polo JR. Downregulation of microRNA-29 by antisense inhibitors and a PPAR-gamma agonist protects against myocardial ischaemia-reperfusion injury. Cardiovasc Res 2010; 87: 535-44.

[41] Zhou L, Wang L, Lu L, Jiang P, Sun H, Wang $\mathrm{H}$. Inhibition of miR-29 by TGF-beta-Smad3 signaling through dual mechanisms promotes transdifferentiation of mouse myoblasts into myofibroblasts. PLoS One 2012; 7: e33766.

[42] Wang S, Aurora AB, Johnson BA, et al. The endothelialspecific microRNA miR-126 governs vascular integrity and angiogenesis. Dev Cell 2008; 15: 261-71.

[43] Nicoli S, Standley C, Walker P, Hurlstone A, Fogarty KE, Lawson ND. MicroRNA-mediated integration of haemodynamics and Vegf signalling during angiogenesis. Nature 2010; 464: 1196-200.

[44] Fish JE, Santoro MM, Morton SU, et al. miR-126 regulates angiogenic signaling and vascular integrity. Dev Cell 2008; 15: 272-84.

[45] van Solingen C, de Boer HC, Bijkerk R, et al. MicroRNA-126 modulates endothelial SDF-1 expression and mobilization of Sca-1(+)/Lin(-) progenitor cells in ischaemia. Cardiovasc Res 2011; 92: 449-55.

[46] Zernecke A, Bidzhekov K, Noels $\mathrm{H}$, et al. Delivery of microRNA-126 by apoptotic bodies induces CXCL12dependent vascular protection. Sci Signal 2009; 2: ra81.

[47] Zampetaki A, Kiechl S, Drozdov I, et al. Plasma microRNA profiling reveals loss of endothelial miR-126 and other microRNAs in type 2 diabetes. Circ Res 2010; 107: 810-7.

[48] Chen Y, Gorski DH. Regulation of angiogenesis through a microRNA (miR-130a) that down-regulates antiangiogenic homeobox genes GAX and HOXA5. Blood 2008; 111: 121726.

[49] Chen JF, Mandel EM, Thomson JM, et al. The role of microRNA-1 and microRNA-133 in skeletal muscle proliferation and differentiation. Nat Genet 2006; 38: 228-33.

[50] Care A, Catalucci D, Felicetti F, et al. MicroRNA-133 controls cardiac hypertrophy. Nat Med 2007; 13: 613-8.

[51] Dong DL, Chen C, Huo R, et al. Reciprocal repression between microRNA-133 and calcineurin regulates cardiac hypertrophy: a novel mechanism for progressive cardiac hypertrophy. Hypertension 2010; 55: 946-52.

[52] Duisters RF, Tijsen AJ, Schroen B, et al. miR-133 and miR30 regulate connective tissue growth factor: implications for a role of microRNAs in myocardial matrix remodeling. Circ Res 2009; 104: 170-8.

[53] Cordes KR, Sheehy NT, White MP, et al. miR-145 and miR143 regulate smooth muscle cell fate and plasticity. Nature 2009; 460: 705-10. 
[54] Boettger T, Beetz N, Kostin S, et al. Acquisition of the contractile phenotype by murine arterial smooth muscle cells depends on the Mir143/145 gene cluster. J Clin Invest 2009; 119: 2634-47.

[55] Xin M, Small EM, Sutherland LB, et al. MicroRNAs miR-143 and miR-145 modulate cytoskeletal dynamics and responsiveness of smooth muscle cells to injury. Genes Dev 2009; 119: 2634-47.

[56] Davis-Dusenbery BN, Chan MC, Reno KE, et al. downregulation of Kruppel-like factor-4 (KLF4) by microRNA$143 / 145$ is critical for modulation of vascular smooth muscle cell phenotype by transforming growth factor-beta and bone morphogenetic protein 4. J Biol Chem 2011; 286: 28097-110.

[57] Boucher JM, Peterson SM, Urs S, Zhang C, Liaw L. The miR-143/145 cluster is a novel transcriptional target of Jagged-1/Notch signaling in vascular smooth muscle cells. J Biol Chem 2011; 286: 28312-21.

[58] Cheng Y, Liu X, Yang J, et al. MicroRNA-145 a novel smooth muscle cell phenotypic marker and modulator controls vascular neointimal lesion formation. Circ Res 2009; 105: 158-66.

[59] Ma X, Ma C, Zheng X. MicroRNA-155 in the pathogenesis of atherosclerosis: a conflicting role? Heart Lung Circ 2013; 22: 811-8.

[60] Martin MM, Buckenberger JA, Jiang J, et al. The human angiotensin II type 1 receptor $+1166 \mathrm{~A} / \mathrm{C}$ polymorphism attenuates microRNA-155 binding. J Biol Chem 2007; 282: 24262-9.

[61] Louafi F, Martinez-Nunez RT, Sanchez-Elsner T. MicroRNA155 targets SMAD2 and modulates the response of macrophages to transforming growth factor- $\beta$. J Biol Chem 2010; 285: 41328-36.

[62] Androulidaki A, Iliopoulos D, Arranz A, et al. The kinase Akt1 controls macrophage response to lipopolysaccharide by regulating microRNAs. Immunity 2009; 31: 220-31.

[63] Nazari-Jahantigh $M$, Wei $Y$, Noels $H$, et al. MicroRNA-155 promotes atherosclerosis by repressing Bcl6 in macrophages. J Clin Invest 2012; 122: 4190-202.

[64] Xu C, Ren G, Cao G, et al. miR-155 regulates immune modulatory properties of mesenchymal stem cells by targeting TAK1-binding protein 2. J Biol Chem 2013; 288: 11074-9.

[65] Zhu N, Zhang D, Chen S, et al. Endothelial enriched microRNAs regulate angiotensin II-induced endothelial inflammation and migration. Atherosclerosis 2011; 215: 28693.

[66] Xu G, Chen J, Jing G, Shalev A. Thioredoxin-interacting protein regulates insulin transcription through microRNA-204. Nat Med 2013; 19: 1141-6.

[67] Caruso P, MacLean MR, Khanin R, et al. Dynamic changes in lung microRNA profiles during the development of pulmonary hypertension due to chronic hypoxia and monocrotaline. Arterioscler Thromb Vasc Biol 2010; 30: 71623.

[68] Courboulin A, Paulin R, Giguere NJ, et al. Role for miR-204 in human pulmonary arterial hypertension. J Exp Med 2011; 208: 535-48.

[69] Paulin R, Meloche J, Jacob MH, Bisserier M, Courboulin A, Bonnet S. Dehydroepiandrosterone inhibits the Src/STAT3 constitutive activation in pulmonary arterial hypertension. Am J Physiol Heart Circ Physiol 2011; 301: H1798-809.

[70] Rossow CF, Minami E, Chase EG, Murry CE, Santana LF. NFATc3-induced reductions in voltage-gated $\mathrm{K}+$ currents after myocardial infarction. Circ Res 2004; 94: 1340-50.

[71] Bonnet S, Rochefort G, Sutendra G, et al. The nuclear factor of activated $T$ cells in pulmonary arterial hypertension can be therapeutically targeted. Proc Natl Acad Sci U S A 2007; 104: 11418-23.

[72] Steiner MK, Syrkina OL, Kolliputi N, Mark EJ, Hales CA, Waxman AB. Interleukin-6 overexpression induces pulmonary hypertension. Circ Res 2009; 104: 236-44.

[73] Mackenzie NC, Staines KA, Zhu D, Genever P, Macrae VE. miRNA-221 and miRNA-222 synergistically function to promote vascular calcification. Cell Biochem Funct 2014; 32: 209-16.

[74] Feng HJ, Ouyang W, Liu JH, et al. Global microRNA profiles and signaling pathways in the development of cardiac hypertrophy. Braz J Med Biol Res 2014; 47: 361-8.

[75] Liu X, Cheng Y, Zhang S, Lin Y, Yang J, Zhang C. A necessary role of miR-221 and miR-222 in vascular smooth muscle cell proliferation and neointimal hyperplasia. Circ Res 2009; 104: 476-87.

[76] Wang C, Wang S, Zhao P, et al. MiR-221 promotes cardiac hypertrophy in vitro through the modulation of p27 expression. J Cell Biochem 2012; 113: 2040-6.

[77] Davis BN, Hilyard AC, Nguyen PH, Lagna G, Hata A. Induction of microRNA-221 by platelet-derived growth factor signaling is critical for modulation of vascular smooth muscle phenotype. J Biol Chem 2009; 284: 3728-38.

[78] Poliseno L, Tuccoli A, Mariani L, et al. MicroRNAs modulate the angiogenic properties of HUVECs. Blood 2006; 108: 3068-71.

[79] Nagalingam RS, Sundaresan NR, Gupta MP, Geenen DL, Solaro RJ, Gupta M. A cardiac-enriched microRNA miR-378 blocks cardiac hypertrophy by targeting Ras signaling. J Biol Chem 2013; 288: 11216-32.

[80] Knezevic I, Patel A, Sundaresan NR, et al. A novel cardiomyocyte-enriched microRNA miR-378 targets insulinlike growth factor 1 receptor: implications in postnatal cardiac remodeling and cell survival. J Biol Chem 2012; 287: 1291326.

[81] Kim SW, Kim HW, Huang W, et al. Cardiac stem cells with electrical stimulation improve ischaemic heart function through regulation of connective tissue growth factor and miR-378. Cardiovasc Res 2013; 100: 241-51.

[82] van Rooij E, Sutherland LB, Qi X, Richardson JA, Hill J, Olson EN. Control of stress-dependent cardiac growth and gene expression by a microRNA. Science 2007; 316: 575-9.

[83] You XY, Huang JH, Liu B, Liu SJ, Zhong Y, Liu SM. HMGA1 is a new target of miR-195 involving isoprenaline-induced cardiomyocyte hypertrophy. Biochemistry (Mosc) 2014; 79: 538-44.

[84] Lanford RE, Hildebrandt-Eriksen ES, Petri A, et al. Therapeutic silencing of microRNA-122 in primates with chronic hepatitis C virus infection. Science 2010; 327: 198201. 